\title{
NEW SINGULAR SOLUTIONS OF PROTTER'S PROBLEM FOR THE 3D WAVE EQUATION
}

\author{
M. K. GRAMMATIKOPOULOS, N. I. POPIVANOV, AND T. P. POPOV
}

Received 10 September 2002

In 1952, for the wave equation, Protter formulated some boundary value problems (BVPs), which are multidimensional analogues of Darboux problems on the plane. $\mathrm{He}$ studied these problems in a $3 \mathrm{D}$ domain $\Omega_{0}$, bounded by two characteristic cones $\Sigma_{1}$ and $\Sigma_{2,0}$ and a plane region $\Sigma_{0}$. What is the situation around these BVPs now after 50 years? It is well known that, for the infinite number of smooth functions in the right-hand side of the equation, these problems do not have classical solutions. Popivanov and Schneider (1995) discovered the reason of this fact for the cases of Dirichlet's or Neumann's conditions on $\Sigma_{0}$. In the present paper, we consider the case of third BVP on $\Sigma_{0}$ and obtain the existence of many singular solutions for the wave equation. Especially, for Protter's problems in $\mathbb{R}^{3}$, it is shown here that for any $n \in \mathbb{N}$ there exists a $C^{n}\left(\bar{\Omega}_{0}\right)$ - right-hand side function, for which the corresponding unique generalized solution belongs to $C^{n}\left(\bar{\Omega}_{0} \backslash O\right)$, but has a strong power-type singularity of order $n$ at the point $O$. This singularity is isolated only at the vertex $O$ of the characteristic cone $\Sigma_{2,0}$ and does not propagate along the cone.

\section{Introduction}

In 1952, at a conference of the American Mathematical Society in New York, Protter introduced some boundary value problems (BVPs) for the 3D wave equation

$$
\square u \equiv u_{x_{1} x_{1}}+u_{x_{2} x_{2}}-u_{t t}=f
$$

in a domain $\Omega_{0} \subset \mathbb{R}^{3}$. These problems are three-dimensional analogous of the Darboux problems (or Cauchy-Goursat problems) on the plane. The simply connected domain

$$
\Omega_{0}:=\left\{\left(x_{1}, x_{2}, t\right): 0<t<\frac{1}{2}, t<\sqrt{x_{1}^{2}+x_{2}^{2}}<1-t\right\}
$$


is bounded by the disk

$$
\Sigma_{0}:=\left\{\left(x_{1}, x_{2}, t\right): t=0, x_{1}^{2}+x_{2}^{2}<1\right\}
$$

centered at the origin $O(0,0,0)$ and by the two characteristic cones of (1.1)

$$
\begin{aligned}
\Sigma_{1}:=\left\{\left(x_{1}, x_{2}, t\right): 0<t<\frac{1}{2}, \sqrt{x_{1}^{2}+x_{2}^{2}}=1-t\right\}, \\
\Sigma_{2,0}:=\left\{\left(x_{1}, x_{2}, t\right): 0<t<\frac{1}{2}, \sqrt{x_{1}^{2}+x_{2}^{2}}=t\right\} .
\end{aligned}
$$

Similar to the plane problems, Protter formulated and studied [24] some 3D problems with data on the noncharacteristic disk $\Sigma_{0}$ and on one of the cones $\Sigma_{1}$ and $\Sigma_{2,0}$. These problems are known now as Portter's problems, defined as follows.

Protter's problems. Find a solution of the wave equation (1.1) in $\Omega_{0}$ with the boundary conditions

(P1) $\left.u\right|_{\Sigma_{0} \cup \Sigma_{1}}=0$,

$\left.\left(\mathrm{P} 1^{*}\right) u\right|_{\Sigma_{0} \cup \Sigma_{2,0}}=0$

(P2) $\left.u\right|_{\Sigma_{1}}=0,\left.u_{t}\right|_{\Sigma_{0}}=0$,

$\left.(\mathrm{P} 2 *) u\right|_{\Sigma_{2,0}}=0,\left.u_{t}\right|_{\Sigma_{0}}=0$.

Substituting the boundary condition on $\Sigma_{0}$ by the third-type condition $\left.\left[u_{t}+\alpha u\right]\right|_{\Sigma_{0}}=$ 0 , we arrive at the following problems.

Problems $\left(P_{\alpha}\right)$ and $\left(P_{\alpha}^{*}\right)$. Find a solution of the wave equation (1.1) in $\Omega_{0}$ which satisfies the boundary conditions

$$
\begin{aligned}
& \left.\left(\mathrm{P}_{\alpha}\right) u\right|_{\Sigma_{1}}=0,\left.\left[u_{t}+\alpha u\right]\right|_{\Sigma_{0} \backslash O}=0, \\
& \left.\left(\mathrm{P}_{\alpha}^{*}\right) u\right|_{\Sigma_{2,0}}=0,\left.\left[u_{t}+\alpha u\right]\right|_{\Sigma_{0} \backslash O}=0,
\end{aligned}
$$

where $\alpha \in C^{1}\left(\bar{\Sigma}_{0} \backslash O\right)$.

The boundary conditions of problem $\left(\mathrm{P} 1^{*}\right)$ (resp., of $\left(\mathrm{P} 2^{*}\right)$ ) are the adjoined boundary conditions to such ones of (P1) (resp., of (P2)) for the wave equation (1.1) in $\Omega_{0}$. Note that Garabedian in [10] proved the uniqueness of a classical solution of problem (P1). For recent results concerning Protter's problems (P1) and (P1*), we refer to [23] and the references therein. For further publications in this area, see $[1,2,8,14,17,18,19,21]$. For problems $\left(\mathrm{P}_{\alpha}\right)$, we refer to [11] and the references therein. In the case of the hyperbolic equation with the wave operator in the main part, which involves either lower-order terms or other type perturbations, problem $\left(\mathrm{P}_{\alpha}\right)$ in $\Omega_{0}$ has been studied by Aldashev in $[1,2,3]$ and by Grammatikopoulos et al. [12]. On the other hand, Ar. B. Bazarbekov and Ak. B. Bazarbekov [5] give another analogue of the classical Darboux problem in the same domain $\Omega_{0}$. Some other statements of Darboux-type problems can be found in $[4,6,16]$ in bounded or unbounded domains different from $\Omega_{0}$.

It is well known that, in contrast to the Darboux problem on the plane, the $3 \mathrm{D}$ problems (P1) and (P2) are not well posed. It is due to the fact that their adjoint homogeneous problems $\left(\mathrm{P} 1^{*}\right)$ and $\left(\mathrm{P} 2^{*}\right)$ have smooth solutions, whose span is infinite-dimensional (see, e.g., Tong [26], Popivanov and Schneider [22], and Khe [18]). 
Now we formulate the following useful lemma, the proof of which is given in Section 2. Lemma 1.1. Let $(\rho, \varphi, t)$ be the polar coordinates in $\mathbb{R}^{3}: x_{1}=\rho \cos \varphi, x_{2}=\rho \sin \varphi$, and $x_{3}=t$. Let $n \in \mathbb{N}, n \geq 4$,

$$
\begin{aligned}
& H_{k}^{n}(\rho, t)=\sum_{i=0}^{k} A_{i}^{k} \frac{t\left(\rho^{2}-t^{2}\right)^{n-3 / 2-k-i}}{\rho^{n-2 i}}, \\
& E_{k}^{n}(\rho, t)=\sum_{i=0}^{k} B_{i}^{k} \frac{\left(\rho^{2}-t^{2}\right)^{n-1 / 2-k-i}}{\rho^{n-2 i}},
\end{aligned}
$$

where

$$
\begin{aligned}
& A_{i}^{k}:=(-1)^{i} \frac{(k-i+1)_{i}(n-1 / 2-k-i)_{i}}{i !(n-i)_{i}}, \\
& B_{i}^{k}:=(-1)^{i} \frac{(k-i+1)_{i}(n+1 / 2-k-i)_{i}}{i !(n-i)_{i}}
\end{aligned}
$$

and $a_{i}:=a(a+1) \cdots(a+i-1)$. Then the functions

$$
V_{k}^{n, 1}(\rho, t, \varphi)=H_{k}^{n}(\rho, t) \sin n \varphi, \quad V_{k}^{n, 2}(\rho, t, \varphi)=H_{k}^{n}(\rho, t) \cos n \varphi,
$$

for $k=0,1, \ldots,[n / 2]-2$, are classical solutions of the homogeneous problem (P1*) (i.e., for $f \equiv 0$ ), and the functions

$$
W_{k}^{n, 1}(\rho, t, \varphi)=E_{k}^{n}(\rho, t) \sin n \varphi, \quad W_{k}^{n, 2}(\rho, t, \varphi)=E_{k}^{n}(\rho, t) \cos n \varphi,
$$

for $k=0,1, \ldots,[(n-1) / 2]-1$, are classical solutions of the homogeneous problem $\left(P 2^{*}\right)$.

A necessary condition for the existence of a classical solution for problem (P2) is the orthogonality of the right-hand side function $f$ to all solutions $W_{k}^{n, i}$ of the homogeneous adjoined problem. In order to avoid an infinite number of necessary conditions in the frame of classical solvability, Popivanov and Schneider in [22, 23] gave definitions of a generalized solution of problem (P2) with an eventual singularity on the characteristic cone $\Sigma_{2,0}$, or only at its vertex $O$. On the other hand, Popivanov and Schneider [23] and Grammatikopoulos et al. [11] proved that for the right-hand side $f=W_{0}^{n, i}$ the corresponding unique generalized solution of problem $\left(\mathrm{P}_{\alpha}\right)$ behaves like $\left(x_{1}^{2}+x_{2}^{2}+t^{2}\right)^{-n / 2}$ around the origin $O$ (for more comments about this subject, we refer to Remarks 1.4 and 1.6). Now we know some solutions, $W_{k}^{n, i}$, of the homogeneous adjoined problem ( $\left.\mathrm{P} 2 *\right)$, and if we take one of these solutions in the right-hand side of (1.1), then we have to expect that the generalized solution of problem $\left(\mathrm{P}_{\alpha}\right)$ will also be singular, possibly with a different power type of singularity. An analogous result, in the case of problem (P1) and functions $V_{k}^{n, i}$, has been proved by Popivanov and Popov in [21]. Having this in mind, here we are looking for some new singular solutions of problem $\left(\mathrm{P}_{\alpha}\right)$, which are different from those found in [11].

In the case of problem $\left(\mathrm{P}_{\alpha}\right)$ with $\alpha(x) \neq 0$, there are only few publications, while for problem $\left(\mathrm{P}_{\alpha}\right)$, concerning the wave equation (1.1), see the results of [11]. Moreover, some results of this type can also be found in Section 3. 
For the homogeneous problem $\left(\mathrm{P}_{\alpha}^{*}\right)$ even for the wave equation (except the case $\alpha \equiv$ 0 , i.e., except problem $\left(\mathrm{P} 2^{*}\right)$ ), we do not know nontrivial solutions analogous to (1.7) and (1.8). In Section 2, we give an approach for finding nontrivial solutions. Relatively, we refer to Khe [18], who found nontrivial solutions for the homogeneous problems $\left(\mathrm{P} 1^{*}\right)$ and $\left(\mathrm{P} 2^{*}\right)$, but in the case of the Euler-Poisson-Darboux equation. These results are closely connected to such ones of Lemma 1.1.

In order to obtain our results, we formulate the following definition of a generalized solution of problem $\left(\mathrm{P}_{\alpha}\right)$ with a possible singularity at $O$.

Definition 1.2. A function $u=u\left(x_{1}, x_{2}, t\right)$ is called a generalized solution of the problem

$$
\left(\mathrm{P}_{\alpha}\right) \square u=f,\left.u\right|_{\Sigma_{1}}=0,\left.\left[u_{t}+\alpha(x) u\right]\right|_{\Sigma_{0}}=0,
$$

in $\Omega_{0}$, if

(1) $u \in C^{1}\left(\bar{\Omega}_{0} \backslash O\right),\left.\left[u_{t}+\alpha(x) u\right]\right|_{\Sigma_{0} \backslash O}=0$, and $\left.u\right|_{\Sigma_{1}}=0$,

(2) the identity

$$
\int_{\Omega_{0}}\left(u_{t} v_{t}-u_{x_{1}} v_{x_{1}}-u_{x_{2}} v_{x_{2}}-f v\right) d x_{1} d x_{2} d t=\int_{\Sigma_{0}} \alpha(x)(u v)(x, 0) d x_{1} d x_{2}
$$

holds for all $v$ in

$$
V_{0}:=\left\{v \in C^{1}\left(\bar{\Omega}_{0}\right):\left.\left[v_{t}+\alpha v\right]\right|_{\Sigma_{0}}=0, v=0 \text { in a neighborhood of } \Sigma_{2,0}\right\} .
$$

Existence and uniqueness results for a generalized solution of problems (P1) and (P2) can be found in [23], while for problem $\left(\mathrm{P}_{\alpha}\right)$, see [11].

In order to deal successfully with the encountered difficulties, as are singularities of generalized solutions on the cone $\Sigma_{2,0}$, we introduce the region

$$
\Omega_{\varepsilon}=\Omega_{0} \cap\{\varrho-t>\varepsilon\}, \quad \varepsilon \in[0,1),
$$

which in polar coordinates becomes

$$
\Omega_{\varepsilon}=\{(\varrho, \varphi, t): t>0,0 \leq \varphi<2 \pi, \varepsilon+t<\varrho<1-t\} .
$$

Note that a generalized solution $u$, which belongs to $C^{1}\left(\bar{\Omega}_{\varepsilon}\right) \cap C^{2}\left(\Omega_{\varepsilon}\right)$ and satisfies the wave equation (1.1) in $\Omega_{\varepsilon}$, is called a classical solution of problem $\left(\mathrm{P}_{\alpha}\right)$ in $\Omega_{\varepsilon}, \varepsilon \in(0,1)$. It should be pointed out that the case $\varepsilon=0$ is totally different from the case $\varepsilon \neq 0$.

This paper is an extension of some results obtained in $[11,12]$ and, besides the introduction, involves two more sections. In Section 2, we formulate the 2D BVPs corresponding to the 3D Protter's problems. Using Riemann functions, we show the way for finding nontrivial solutions. For the same goal, we consider functions orthogonal to the Legendre one and formulate some open questions for finding more functions of this type in the frame of nontrivial solutions of problems $\left(\mathrm{P} 1^{*}\right),\left(\mathrm{P} 2^{*}\right)$, and $\left(\mathrm{P}_{\alpha}^{*}\right)$. Also, using the results of Sections 1 and 2, in Section 3, we study the existence of a singular generalized solution of $3 \mathrm{D}$ problem $\left(\mathrm{P}_{\alpha}\right)$. To investigate the behavior of such singular solutions, we need some information about them. In Theorem 3.1, we state a maximum principle for the singular generalized solution of $2 \mathrm{D}$ problem $\left(\mathrm{P}_{\alpha, 2}\right)$, corresponding to problem $\left(\mathrm{P}_{\alpha}\right)$ 
in $\Omega_{0}$. This solution is a classical one in each domain $\Omega_{\varepsilon}, \varepsilon \in(0,1)$. Note that this maximum principle can be applied even in the cases where the right-hand side changes its sign in the domain. (Theorem 1.3 deals exactly with this special situation.) Other maximum principles can be found in $[6,25]$. Using information of this kind, we present singular generalized solutions which are smooth enough away from the point $O$, while at the point $O$, they have power-type singularity. More precisely, in Section 3, we prove the following theorem.

Theorem 1.3. Let $\alpha=\alpha(\rho) \in C^{\infty}(0,1] \cap C[0,1]$ and let $\alpha(\rho) \geq 0$ be an arbitrary function. Then, for each $n \in \mathbb{N}, n \geq 4$, there exists a function $f_{n} \in C^{n-3}\left(\bar{\Omega}_{0}\right) \cap C^{\infty}\left(\Omega_{0}\right)$, for which the corresponding unique generalized solution $u_{n}$ of problem $\left(P_{\alpha}\right)$ belongs to $C^{n-1}\left(\bar{\Omega}_{0} \backslash O\right)$ and satisfies the estimates

$$
\begin{aligned}
& \left|u_{n}\left(x_{1}, x_{2},|x|\right)\right| \geq \frac{1}{2}\left|u_{n}\left(2 x_{1}, 2 x_{2}, 0\right)\right|+|x|^{-(n-2)}\left|\cos n\left(\arctan \frac{x_{2}}{x_{1}}\right)\right|, \\
& \left|u_{n}\left(x_{1}, x_{2}, \frac{1-\tau n_{1}}{1+\tau n_{1}}|x|\right)\right| \geq|x|^{-(n-2)}\left|\cos n\left(\arctan \frac{x_{2}}{x_{1}}\right)\right|, \quad 0 \leq \tau \leq 1,
\end{aligned}
$$

where the constant $n_{1} \in(0,1)$ depends only on $n$.

Remark 1.4. For the right-hand side of the wave equation equals $W_{0}^{n, 2}$, the exact behavior of the corresponding singular solution $u_{n}\left(x_{1}, x_{2}, t\right)$ around the origin $O$ is $\left(x_{1}^{2}+\right.$ $\left.x_{2}^{2}+t^{2}\right)^{-n / 2} \cos n\left(\arctan x_{2} / x_{1}\right)$ (see [11, 12]), while for the right-hand side equals $W_{1}^{n, 2}=\partial^{2} / \partial t^{2}\left\{W_{0}^{n, 2}\right\}$, the singularities are at least of type $\left(x_{1}^{2}+x_{2}^{2}+t^{2}\right)^{-(n-2) / 2} \cos$ $n\left(\arctan x_{2} / x_{1}\right)$ (see Theorem 1.3 ). The following open question arises: is this the exact type of singularity or not? If the last case is true, it would be possible, using an appropriate linear combination of both right-hand sides, to find a solution of the last lower-type singularity. Then the result of this kind could give an answer to Open Question (1).

Remark 1.5. It is interesting that for any parameter $\alpha(x) \geq 0$, involved in the boundary condition $\left(\mathrm{P}_{\alpha}\right)$ on $\Sigma_{0}$, there are infinitely many singular solutions of the wave equation. Note that all these solutions have strong singularities at the vertex $O$ of the cone $\Sigma_{2,0}$. These singularities of generalized solutions do not propagate in the direction of the bicharacteristics on the characteristic cone. It is traditionally assumed that the wave equation with right-hand side sufficiently smooth in $\bar{\Omega}_{0}$ cannot have a solution with an isolated singular point. For results concerning the propagation of singularities for secondorder operators, see Hörmander [13, Chapter 24.5]. For some related results in the case of the plane Darboux problem, see [20].

Remark 1.6. Considering problems (P1) and (P2), Popivanov and Schneider [22] announced the existence of singular solutions for both wave and degenerate hyperbolic equations. First a priori estimates for singular solutions of Protter's problems (P1) and (P2), concerning the wave equation in $\mathbb{R}^{3}$, were obtained in [23]. In [1], Aldashev mentioned the results of [22] and, for the case of the wave equation in $\mathbb{R}^{m+1}$, showed that there exist solutions of problem (P1) (resp., (P2)) in the domain $\Omega_{\varepsilon}$, which grow up on the cone $\Sigma_{2, \varepsilon}$ like $\varepsilon^{-(n+m-2)}$ (resp., $\varepsilon^{-(n+m-1)}$ ), and the cone $\Sigma_{2, \varepsilon}:=\{\varrho=t+\varepsilon\}$ approximates $\Sigma_{2,0}$ when $\varepsilon \rightarrow 0$. It is obvious that, for $m=2$, these results can be compared to 
the estimates of [11]. Finally, we point out that in the case of an equation which involves the wave operator and nonzero lower-order terms, Karatoprakliev [15] obtained a priori estimates, but only for the enough smooth solutions of problem (P1) in $\Omega_{0}$.

We fix the right-hand side as a trigonometric polynomial of the order $l$ :

$$
f\left(x_{1}, x_{2}, t\right)=\sum_{n=2}^{l}\left\{f_{n}^{1}(t, \rho) \cos n \varphi+f_{n}^{2}(t, \rho) \sin n \varphi\right\} .
$$

We already know that the corresponding solution $u\left(x_{1}, x_{2}, t\right)$ may have behavior of type $\left(x_{1}^{2}+x_{2}^{2}+t^{2}\right)^{-l / 2}$ at the point $O$. We conclude this section with the following questions.

Open Questions. (1) Find the exact behavior of all singular solutions at the point $O$, which differ from those of Theorem 1.3. In other words,

(i) are there generalized solutions for the right-hand side (1.14) with a higher order of singularity, for example, of the form $\left(x_{1}^{2}+x_{2}^{2}+t^{2}\right)^{-k-l / 2}, k>0$ ?

(ii) are there generalized solutions for the right-hand side (1.14) with a lower order of singularity, for example, of the form $\left(x_{1}^{2}+x_{2}^{2}+t^{2}\right)^{k-l / 2}, k>0$ ?

(2) Find appropriate conditions for the function $f$ under which problem $\left(\mathrm{P}_{\alpha}\right)$ has only classical solutions. We do not know any kind of such results even for problem (P2).

(3) From the a priori estimates, obtained in [11], for all solutions of problem $\left(\mathrm{P}_{\alpha}\right)$, including singular ones, it follows that, as $\rho \rightarrow 0$, none of these solutions can grow up faster than the exponential one. The arising question is: are there singular solutions of problem $\left(\mathrm{P}_{\alpha}\right)$ with exponential growth as $\rho \rightarrow 0$ or any such solution is of polynomial growth less than or equal to $\left(x_{1}^{2}+x_{2}^{2}+t^{2}\right)^{-l / 2}$ ?

(4) Why there appear singularities for smooth right-hand side, even for the wave equation? Can we explain this phenomenon numerically?

In the case of problem (P1), the answers to Open Questions (1), (2), and (3) can be found in [21].

\section{Nontrivial solutions for the homogeneous problems $\left(\mathbf{P} 1^{*}\right),\left(\mathrm{P} 2^{*}\right)$, and $\left(\mathrm{P}_{\alpha}^{*}\right)$}

Suppose that the right-hand side $f$ of the wave equation is of the form

$$
f(\rho, t, \varphi)=f_{n}^{1}(\rho, t) \cos n \varphi+f_{n}^{2}(\rho, t) \sin n \varphi, \quad n \in \mathbb{N} .
$$

Then we are seeking solutions of the wave equation of the same form

$$
u(\rho, t, \varphi)=u_{n}^{1}(\rho, t) \cos n \varphi+u_{n}^{2}(\rho, t) \sin n \varphi,
$$

and due to this fact, the wave equation reduces to

$$
\left(u_{n}\right)_{\rho \rho}+\frac{1}{\rho}\left(u_{n}\right)_{\rho}-\left(u_{n}\right)_{t t}-\frac{n^{2}}{\rho^{2}} u_{n}=f_{n}
$$

in $G_{0}=\{0<t<1 / 2 ; t<\rho<1-t\} \subset \mathbb{R}^{2}$. 
Now introduce the new coordinates $x=(\rho+t) / 2, y=(\rho-t) / 2$ and set

$$
v(x, y)=\rho^{1 / 2} u_{n}(\rho, t), \quad g(x, y)=\rho^{1 / 2} f_{n}(\rho, t) .
$$

Then, denoting $v=n-(1 / 2)$, problems $\left(\mathrm{P} 1^{*}\right),\left(\mathrm{P} 2^{*}\right)$, and $\left(\mathrm{P}_{\alpha}^{*}\right)$ transform into the following problems.

Problems (P31), (P32), and $\left(P 3_{\alpha}\right)$. Find a solution $v(x, y)$ of the equation

$$
v_{x y}-\frac{\nu(\nu+1)}{(x+y)^{2}} v=g
$$

in the domain $D=\{0<x<1 / 2 ; 0<y<x\}$ with the following corresponding boundary conditions:

(P31) $v(x, x)=0, x \in(0,1 / 2)$ and $v(1 / 2, y)=0, y \in(0,1 / 2)$,

(P32) $\left(v_{y}-v_{x}\right)(x, x)=0, x \in(0,1 / 2)$ and $v(1 / 2, y)=0, y \in(0,1 / 2)$,

$\left(\mathrm{P} 3_{\alpha}\right)\left(v_{y}-v_{x}\right)(x, x)-\alpha(x) v(x, x)=0, x \in(0,1 / 2)$ and $v(1 / 2, y)=0, y \in(0,1 / 2)$.

A basic tool for our treatment of problems (P3) is the Legendre functions $P_{\nu}$ (for more information, see [9]). Note that the function

$$
R\left(x_{1}, y_{1} ; x, y\right)=P_{\nu}\left(\frac{(x-y)\left(x_{1}-y_{1}\right)+2 x_{1} y_{1}+2 x y}{\left(x_{1}+y_{1}\right)(x+y)}\right)
$$

is a Riemann one for (2.5) (see Copson [7]), that is, with respect to the variables $\left(x_{1}, y_{1}\right)$, it is a solution of (2.5) with $g=0$, and

$$
R\left(x, y_{1} ; x, y\right)=1, \quad R\left(x_{1}, y ; x, y\right)=1 .
$$

Therefore, we can construct the function $u(x, y)$ in the following way. Integrating (2.5) over the characteristic triangle $\triangle$ with vertices $M(x, y) \in D, P(y, y)$, and $Q(x, x)$, and using the properties (2.7) of the Riemann function, we see that

$$
\begin{aligned}
\iint_{\triangle} R & \left(x_{1}, y_{1} ; x, y\right) g\left(x_{1}, y_{1}\right) d x_{1} d y_{1} \\
= & \int_{y}^{x}\left[R\left(x_{1}, x_{1} ; x, y\right) v_{x_{1}}\left(x_{1}, x_{1}\right)-R\left(x_{1}, y ; x, y\right) v_{x_{1}}\left(x_{1}, y\right)\right] d x_{1} \\
& \quad-\int_{y}^{x}\left[R_{y_{1}}\left(x, y_{1} ; x, y\right) v\left(x, y_{1}\right)-R_{y_{1}}\left(y_{1}, y_{1} ; x, y\right) v\left(y_{1}, y_{1}\right)\right] d y_{1} \\
= & \int_{y}^{x}\left[R\left(x_{1}, x_{1} ; x, y\right) v_{x_{1}}\left(x_{1}, x_{1}\right)+R_{y_{1}}\left(x_{1}, x_{1} ; x, y\right) v\left(x_{1}, x_{1}\right)\right] d x_{1} \\
& -v(x, y)+v(y, y) .
\end{aligned}
$$

Hence

$$
\begin{aligned}
v(x, y)= & v(y, y)+\int_{y}^{x}\left[R\left(x_{1}, x_{1} ; x, y\right) v_{x_{1}}\left(x_{1}, x_{1}\right)+R_{y_{1}}\left(x_{1}, x_{1} ; x, y\right) v\left(x_{1}, x_{1}\right)\right] d x_{1} \\
& -\iint_{\triangle} R\left(x_{1}, y_{1} ; x, y\right) g\left(x_{1}, y_{1}\right) d x_{1} d y_{1} .
\end{aligned}
$$


322 New singular solutions of Protter's problem

In the case of $g=0$, we obtain

$$
\begin{aligned}
v(x, y)=v(y, y)+\int_{y}^{x} & {\left[P_{\nu}\left(\frac{x_{1}^{2}+x y}{x_{1}(x+y)}\right) v_{x_{1}}\left(x_{1}, x_{1}\right)\right.} \\
& \left.+P_{\nu}^{\prime}\left(\frac{x_{1}^{2}+x y}{x_{1}(x+y)}\right) \frac{\left(x_{1}-x\right)\left(x_{1}+y\right)}{2 x_{1}^{2}(x+y)} v\left(x_{1}, x_{1}\right)\right] d x_{1} .
\end{aligned}
$$

Using the condition $v(x, 0)=0$, finally we find that

$$
\begin{aligned}
0 & =\int_{0}^{x} P_{\nu}\left(\frac{x_{1}}{x}\right) v_{x_{1}}\left(x_{1}, x_{1}\right)+P_{\nu}^{\prime}\left(\frac{x_{1}}{x}\right) \frac{\left(x_{1}-x\right)}{2 x_{1} x} v\left(x_{1}, x_{1}\right) d x_{1} \\
& =\int_{0}^{x} P_{\nu}\left(\frac{x_{1}}{x}\right)\left\{v_{x_{1}}\left(x_{1}, x_{1}\right)-\frac{\partial}{\partial x_{1}}\left[v\left(x_{1}, x_{1}\right) \frac{\left(x_{1}-x\right)}{2 x_{1}}\right]\right\} d x_{1}
\end{aligned}
$$

if we suppose, in addition, that $\lim t^{-1} v(t, t)=0, t \rightarrow+0$. Thus,

$$
\int_{0}^{1} P_{\nu}(t)\left\{\frac{t+1}{t} v_{x}(t x, t x)+\frac{1-t}{t} v_{y}(t x, t x)-\frac{1}{x t^{2}} v(t x, t x)\right\} d t=0
$$

Suppose that there exist two functions $\psi$ and $\psi_{1}$ such that

$$
\psi(t) \psi_{1}(x)=\frac{t+1}{t} v_{x}(t x, t x)+\frac{1-t}{t} v_{y}(t x, t x)-\frac{1}{x t^{2}} v(t x, t x) .
$$

Then we are looking for a solution $\psi(t)$ of the equation

$$
\int_{0}^{1} P_{\nu}(t) \psi(t) d t=0
$$

Now we are ready to formulate the following useful lemma.

Lemma 2.1. The following identity holds:

$$
\int_{0}^{1} t^{p} P_{\nu}(t) d t=0, \quad p=v-2, v-4, \ldots ; p>-1
$$

Proof. As known, the Legendre functions $P_{\nu}(t)$ are solutions of the Legendre differential equation

$$
\left(1-t^{2}\right) z^{\prime \prime}-2 t z^{\prime}+\nu(\nu+1) z=0 .
$$


Using this fact, we see that

$$
\begin{aligned}
\nu(\nu+1) \int_{0}^{1} t^{p} P_{\nu}(t) d t & =\int_{0}^{1} t^{p}\left[\left(t^{2}-1\right) P_{\nu}^{\prime}(t)\right]^{\prime} d t \\
& =-p \int_{0}^{1}\left(t^{p+1}-t^{p-1}\right) P_{\nu}^{\prime}(t) d t \\
& =p \int_{0}^{1}\left(t^{p+1}-t^{p-1}\right) P_{\nu}^{\prime}(t) d t \\
& =p \int_{0}^{1}\left[(p+1) t^{p}-(p-1) t^{p-2}\right] P_{\nu}(t) d t
\end{aligned}
$$

if $p>1$. This means that

$$
[\nu(\nu+1)-p(p+1)] \int_{0}^{1} t^{p} P_{\nu}(t) d t=-p(p-1) \int_{0}^{1} t^{p-2} P_{\nu}(t) d t, \quad p>1 .
$$

Since, for $p=v$, the left-hand side here is zero, clearly

$$
\int_{0}^{1} t^{\nu-2} P_{\nu}(t) d t=0
$$

Using this fact and (2.18) with $p=v-2$, we conclude that

$$
\int_{0}^{1} t^{\nu-4} P_{\nu}(t) d t=0, \quad \text { if } \nu-2>1
$$

and so the proof of the lemma follows by induction.

Since, in our case, $v=n-1 / 2$, returning to problems $\left(\mathrm{P} 1^{*}\right),\left(\mathrm{P} 2^{*}\right)$, and $\left(\mathrm{P}_{\alpha}^{*}\right)$, we remark that, for each of these problems, we have the following conclusions.

Problem $\left(P 1^{*}\right)$. On the line $\{y=x\}$, we have the condition $v(x, x)=0$. Thus, $\left(v_{x}+v_{y}\right)(x$, $x)=0$ and (2.13) becomes $\psi(t) \psi_{1}(x)=2 v_{x}(t x, t x)$. It follows that in this case, by Lemma 2.1 , possible solutions are the functions

$$
v(x, x)=0, \quad v_{x}(x, x)=x^{p},
$$

where $p=n-5 / 2, n-9 / 2, \ldots, 1 / 2$, if $n$ is an odd number, or $p=n-5 / 2, n-9 / 2, \ldots,-1 / 2$, if $n$ is an even number. Thus, the solution $v(x, y)$ of the homogeneous problem $\left(\mathrm{P} 1^{*}\right)$ is explicitly found by (2.10) with values of $v$ and $v_{x}$ on $\{y=x\}$ given by (2.21).

Problem (P2*). In this case, for $y=x$, we have $\left(v_{x}-v_{y}\right)(x, x)=0$. Denote $h(x):=v(x, x)$, then $h^{\prime}(x)=v_{x}(x, x)+v_{y}(x, x)$. Hence, we see that $v_{x}=v_{y}=h^{\prime} / 2$ and (2.13) becomes

$$
\psi\left(\frac{z}{x}\right) \psi_{1}(x)=\frac{x}{z} h^{\prime}(z)-\frac{x}{z^{2}} h(z)=x\left(\frac{h(z)}{z}\right)^{\prime} .
$$

By Lemma 2.1, possible solutions of the above equation are the functions

$$
v(x, x)=x^{p}, \quad v_{x}(x, x)=\frac{p x^{p-1}}{2}
$$


where $p=n-1 / 2, n-5 / 2, \ldots, 5 / 2$, if $n$ is an odd number, or $p=n-1 / 2, n-5 / 2, \ldots, 3 / 2$, if $n$ is an even number. The corresponding solution $v(x, y)$ of the homogeneous problem $\left(\mathrm{P} 2^{*}\right)$ is found again by (2.10) with values of $v(x, x)$ and $v_{x}(x, x)$ given by $(2.23)$.

Problem $\left(P_{\alpha}^{*}\right)$. Denote $h(x):=v(x, x)$. Then together with the condition on the line $\{y=x\}$, we see that

$$
h^{\prime}(x)=v_{x}(x, x)+v_{y}(x, x), \quad v_{y}(x, x)-v_{x}(x, x)-\alpha(x) v(x, x)=0,
$$

from where we have $v_{y}=\left(h^{\prime}+\alpha h\right) / 2$ and $v_{x}=\left(h^{\prime}-\alpha h\right) / 2$. In this case, $(2.13)$ becomes

$$
\psi\left(\frac{z}{x}\right) \psi_{1}(x)=x\left(\frac{h(z)}{z}\right)^{\prime}-\alpha(z) h(z)
$$

If $\alpha(z)$ is not identically zero, it is not obvious whether there are some nontrivial solutions of problem $\left(\mathrm{P}_{\alpha}^{*}\right)$ or not.

Open problems. (1) Find a solution $\psi(t)$ of (2.14), different from those of (2.15), which gives a new nontrivial solution of problem $\left(\mathrm{P}^{*}\right)$ or $\left(\mathrm{P} 2^{*}\right)$.

(2) Using the way described above, find nontrivial solutions of problem $\left(\mathrm{P}_{\alpha}^{*}\right)$, when $\alpha(x)$ is a nonzero function.

The representation (2.10), together with (2.21) and (2.23), gives us exact formulae for the solution of the homogeneous problems $\left(\mathrm{P} 1^{*}\right)$ and $\left(\mathrm{P} 2^{*}\right)$. Using Lemma 1.1, we obtain a different representation of the same solutions. The solutions $V_{0}^{n, i}$ and $W_{0}^{n, i}$ were found by Popivanov and Schneider, while the functions $H_{k}^{n}$ and $E_{k}^{n}$ can be found in [18] with a different presentation, where they are defined by using the Gauss hypergeometric function.

The following result implies Lemma 1.1.

Lemma 2.2. The representations

$$
\begin{aligned}
& \frac{\partial}{\partial t} H_{k}^{n}(\rho, t)=2(n-k-1) E_{k+1}^{n}(\rho, t), \\
& \frac{\partial}{\partial t} E_{k}^{n}(\rho, t)=-2\left(n-k-\frac{1}{2}\right) H_{k}^{n}(\rho, t)
\end{aligned}
$$

hold, where $H_{k}^{n}$ and $E_{k}^{n}$ represent derivatives of $E_{0}^{n}(\rho, t)$ with respect to $t$, that is,

$$
\begin{aligned}
& H_{k}^{n}(\rho, t)=\frac{(-1)^{k+1}}{(2 n-2 k-1)_{2 k+1}}\left(\frac{\partial}{\partial t}\right)^{2 k+1}\left(\frac{\left(\rho^{2}-t^{2}\right)^{n-1 / 2}}{\rho^{n}}\right) \\
& E_{k}^{n}(\rho, t)=\frac{(-1)^{k}}{(2 n-2 k)_{2 k}}\left(\frac{\partial}{\partial t}\right)^{2 k}\left(\frac{\left(\rho^{2}-t^{2}\right)^{n-1 / 2}}{\rho^{n}}\right)
\end{aligned}
$$

Proof. It is enough to check directly formulae (2.26) and (2.27). 
Proof of Lemma 1.1. We already know (see [23]) that $V_{0}^{n, i}$ and $W_{0}^{n, i}(i=1,2)$ are solutions of the wave equation (1.1). Using formulae (2.26) and (2.27), we conclude that $V_{k}^{n, i}$ and $W_{k}^{n, i}$ are also solutions of the wave equation. Thus, the functions $\rho^{1 / 2} H_{k}^{n}(t, \rho)$ and $\rho^{1 / 2} E_{k}^{n}(t, \rho)$ are solutions of the $2 \mathrm{D}$ equation (2.5). It is easy to see directly that

$$
\frac{\partial\left(\rho^{1 / 2} E_{k}^{n}\right)}{\partial t}(\rho, 0)=0, \quad\left(\rho^{1 / 2} E_{k}^{n}\right)(\rho, 0)=\rho^{n-2 k-1 / 2} \sum_{i=0}^{k} A_{i}^{k} .
$$

These Cauchy conditions on $\{x=y\}$ (i.e., on $\{t=0\}$ ) coincide with the conditions of (2.23) for $p=n-2 k-1 / 2$ with the accuracy of a multiplicative constant. Moreover, because of the uniqueness of the solution of Cauchy problem for $(2.5)$, the function $v(x, y)$ defined by (2.10), together with the conditions of (2.23) for $p=n-2 k-1 / 2$, coincides with the function $\left(\sum_{i=0}^{k} A_{i}^{k}\right)^{-1} \rho^{1 / 2} E_{k}^{n}(\rho, t)$.

\section{New singular solutions of problem $\left(\mathbf{P}_{\alpha}\right)$}

We are seeking a generalized solution of $\mathrm{BVP}\left(\mathrm{P}_{\alpha}\right)$ for the wave equation

$$
\square u=\frac{1}{\varrho}\left(\varrho u_{\varrho}\right)_{\varrho}+\frac{1}{\varrho^{2}} u_{\varphi \varphi}-u_{t t}=f(\varrho, \varphi, t)
$$

which has some power type of singularity at the origin $O$. While in $[11,23]$ the function $W_{0}^{n, i}(\rho, t, \varphi)$ has been used systematically as the right-hand side function, we will try to use here, for the same reason, the function $W_{1}^{n, i}(\rho, t, \varphi)$. Due to the fact that the function $E_{1}^{n}(\rho, t)$ changes its sign inside the domain, the appearing situation causes some complications. Note first that, by Lemma 1.1, the functions

$$
W_{1}^{n, 2}(\varrho, \varphi, t)=\left\{\frac{\left(\varrho^{2}-t^{2}\right)^{n-3 / 2}}{\varrho^{n}}-\frac{(n-3 / 2)}{(n-1)} \frac{\left(\varrho^{2}-t^{2}\right)^{n-5 / 2}}{\varrho^{n-2}}\right\} \cos n \varphi, \quad n \geq 4
$$

with $W_{1}^{n, 2} \in C^{n-3}\left(\bar{\Omega}_{0}\right)$, are classical solutions of problem $\left(\mathrm{P}_{\alpha}^{*}\right)$ when $\alpha \equiv 0$.

To prove Theorem 1.3, consider now the special case of problem $\left(\mathrm{P}_{\alpha}\right)$ :

$$
\begin{gathered}
\square u=\frac{1}{\varrho}\left(\varrho u_{\varrho}\right)_{\varrho}+\frac{1}{\varrho^{2}} u_{\varphi \varphi}-u_{t t}=W_{1}^{n, 2}(\varrho, \varphi, t) \quad \text { in } \Omega_{0}, \\
\left.u\right|_{\Sigma_{1}}=0,\left.\quad\left[u_{t}+\alpha(\varrho) u\right]\right|_{\Sigma_{0} \backslash O}=0 .
\end{gathered}
$$

Theorem 5.1 of [11] declares that problem (3.3), (3.4) has at most one generalized solution. On the other hand, by [11, Theorem 5.2], we know that for this right-hand side there exists a generalized solution in $\Omega_{0}$ of the form

$$
u_{n}(\varrho, \varphi, t)=u_{n}^{(1)}(\varrho, t) \cos n \varphi \in C^{n-1}\left(\bar{\Omega}_{0} \backslash O\right),
$$

which is a classical solution in $\Omega_{\varepsilon}, \varepsilon \in(0,1)$. By introducing a new function

$$
u^{(2)}(\varrho, t)=\varrho^{1 / 2} u^{(1)}(\varrho, t)
$$


we transform (3.3) into the equation

$$
u_{\varrho \varrho}^{(2)}-u_{t t}^{(2)}-\frac{4 n^{2}-1}{4 \varrho^{2}} u^{(2)}=\varrho^{1 / 2} E_{1}^{n}(\varrho, t),
$$

with the string operator in the main part. The domain, corresponding to $\Omega_{\varepsilon}$ in this case, is

$$
G_{\varepsilon}=\{(\varrho, t): t>0, \varepsilon+t<\varrho<1-t\}
$$

In order to use directly the results of [11], we introduce the new coordinates

$$
\xi=1-\varrho-t, \quad \eta=1-\varrho+t
$$

and transform the singular point $O$ into the point $(1,1)$.

From (3.7), we derive that

$$
U_{\xi \eta}-\frac{4 n^{2}-1}{4(2-\xi-\eta)^{2}} U=\frac{1}{4 \sqrt{2}}(2-\eta-\xi)^{1 / 2} F(\xi, \eta)
$$

in $D_{\varepsilon}=\{(\xi, \eta): 0<\xi<\eta<1-\varepsilon\}$, where

$$
U(\xi, \eta)=u^{(2)}(\rho(\xi, \eta), t(\xi, \eta)), \quad F(\xi, \eta)=E_{1}^{n}(\rho(\xi, \eta), t(\xi, \eta))
$$

In order to investigate the smoothness or the singularity of a solution for the original 3D problem $\left(\mathrm{P}_{\alpha}\right)$ on $\Sigma_{2,0}$, we are seeking a classical solution of the corresponding $2 \mathrm{D}$ problem $\left(\mathrm{P}_{\alpha, 2}\right)$, not only in the domain $D_{\varepsilon}$ but also in the domain

$$
D_{\varepsilon}^{(1)}:=\{(\xi, \eta): 0<\xi<\eta<1,0<\xi<1-\varepsilon\}, \quad \varepsilon>0 .
$$

Clearly, $D_{\varepsilon} \subset D_{\varepsilon}^{(1)}$. Thus, we arrive at the Goursat-Darboux problem.

Problem $\left(P_{\alpha, 2}\right)$. Find a solution of the following BVP:

$$
\begin{gathered}
U_{\xi \eta}-c(\xi, \eta) U=g(\xi, \eta) \quad \text { in } D_{\varepsilon}^{(1)}, \\
U(0, \eta)=0,\left.\quad\left[U_{\eta}-U_{\xi}+\alpha(1-\xi) U\right]\right|_{\eta=\xi}=0 .
\end{gathered}
$$

Here, the coefficients $c(\xi, \eta)$ and $g(\xi, \eta)$ are defined by

$$
\begin{aligned}
& c(\xi, \eta)=\frac{4 n^{2}-1}{4(2-\eta-\xi)^{2}} \in C^{\infty}\left(\bar{D}_{\varepsilon}^{(1)}\right), \quad n \geq 4, \varepsilon>0, \\
& g(\xi, \eta)=2^{n-(5 / 2)}\left\{\frac{[(1-\xi)(1-\eta)]^{n-3 / 2}}{(2-\eta-\xi)^{n-1 / 2}}-\frac{(n-3 / 2)}{4(n-1)} \frac{[(1-\xi)(1-\eta)]^{n-5 / 2}}{(2-\eta-\xi)^{n-5 / 2}}\right\},
\end{aligned}
$$


where $g \in C^{n-3}\left(\bar{D}_{\varepsilon}^{(1)}\right)$. In this case, it is obvious that $c(\xi, \eta) \geq 0$ in $\bar{D}_{0} \backslash(1,1)$, but the function $g(\xi, \eta)$ is not nonnegative in $D_{0}$.

Note that, according to [11], solving problem $\left(\mathrm{P}_{\alpha, 2}\right)$ is equivalent to solving the following integral equation:

$$
\begin{aligned}
U\left(\xi_{0}, \eta_{0}\right)= & \int_{0}^{\xi_{0}} \int_{\xi_{0}}^{\eta_{0}}[g(\xi, \eta)+c(\xi, \eta) U(\xi, \eta)] d \eta d \xi \\
& +2 \int_{0}^{\xi_{0}} \int_{0}^{\eta}[g(\xi, \eta)+c(\xi, \eta) U(\xi, \eta)] d \xi d \eta \\
& +\int_{0}^{\xi_{0}} \alpha(1-\xi) U(\xi, \xi) d \xi \quad \text { for }\left(\xi_{0}, \eta_{0}\right) \in \bar{D}_{\varepsilon}^{(1)}
\end{aligned}
$$

For this reason, we define (see [11]) the following sequence of successive approximations $U^{(m)}$ :

$$
\begin{aligned}
U^{(m+1)}\left(\xi_{0}, \eta_{0}\right)= & \int_{0}^{\xi_{0}} \int_{\xi_{0}}^{\eta_{0}}\left[g(\xi, \eta)+c(\xi, \eta) U^{(m)}(\xi, \eta)\right] d \eta d \xi \\
& +2 \int_{0}^{\xi_{0}} \int_{0}^{\eta}\left[g(\xi, \eta)+c(\xi, \eta) U^{(m)}(\xi, \eta)\right] d \xi d \eta \\
& +\int_{0}^{\xi_{0}} \alpha(1-\xi) U^{(m)}(\xi, \xi) d \xi, \quad\left(\xi_{0}, \eta_{0}\right) \in \bar{D}_{\varepsilon}^{(1)}, \\
U^{(0)}\left(\xi_{0}, \eta_{0}\right)= & 0 \quad \text { in } D_{\varepsilon}^{1} .
\end{aligned}
$$

In [11], the uniform convergence of $U^{(m)}$ in each domain $D_{\varepsilon}^{(1)}, \varepsilon>0$, has been proved. To use this fact here, we now formulate the following maximum principle, which is very important for the investigation of the singularity of a generalized solution of problem $\left(\mathrm{P}_{\alpha}\right)$.

Theorem 3.1 (maximum principle). Let $c(\xi, \eta), g(\xi, \eta) \in C\left(\bar{D}_{\varepsilon}^{(1)}\right)$, let $c(\xi, \eta) \geq 0$ in $\bar{D}_{\varepsilon}^{(1)}$, let $\alpha(\xi) \geq 0$ for $0 \leq \xi \leq 1$, and

(a) let

$$
\int_{0}^{\xi_{0}} \int_{\xi_{0}}^{\eta_{0}} g(\xi, \eta) d \eta d \xi+2 \int_{0}^{\xi_{0}} \int_{0}^{\eta} g(\xi, \eta) d \xi d \eta \geq 0 \quad \text { in } \bar{D}_{\varepsilon}^{(1)} .
$$

Then, for the solution $U(\xi, \eta)$ of problem (3.13), it holds that

$$
U(\xi, \eta) \geq 0 \quad \text { in } \bar{D}_{\varepsilon}^{(1)} .
$$

(b) If

$$
\int_{0}^{\xi_{0}} g\left(\xi, \eta_{0}\right) d \xi \geq 0 \quad \text { in } \bar{D}_{\varepsilon}^{(1)}
$$


328 New singular solutions of Protter's problem

then

$$
U(\xi, \eta) \geq 0, \quad U_{\eta}(\xi, \eta) \geq 0 \quad \text { for }(\xi, \eta) \in \bar{D}_{\varepsilon}^{(1)}
$$

(c) If $g(\xi, \eta) \geq 0$ in $\bar{D}_{\varepsilon}^{(1)}$, then

$$
U(\xi, \eta) \geq 0, \quad U_{\eta}(\xi, \eta) \geq 0, \quad U_{\xi}(\xi, \eta) \geq 0 \quad \text { in } \bar{D}_{\varepsilon}^{(1)}
$$

Remark 3.2. Other variants of this maximum principle can be found in $[11,12]$. In the cases which we consider below, the conditions of $[11,12]$ are not satisfied. For example, there are subdomains of $D_{\varepsilon}^{(1)}$ where $E_{1}^{n}<0$.

Proof of Theorem 3.1. (a) Condition (3.18) says that for the first approximation $U^{(1)}$ of the sequence $(3.17)$, we directly have $U^{(1)}\left(\xi_{0}, \eta_{0}\right) \geq 0$. Suppose that $\left(U^{(m)}-U^{(m-1)}\right)\left(\xi_{0}, \eta_{0}\right) \geq$ 0 for some $m \in \mathbb{N}$. Then

$$
\begin{aligned}
\left(U^{(m+1)}-U^{(m)}\right)\left(\xi_{0}, \eta_{0}\right)= & \int_{0}^{\xi_{0}} \int_{\xi_{0}}^{\eta_{0}} c(\xi, \eta)\left(U^{(m)}-U^{(m-1)}\right)(\xi, \eta) d \eta d \xi \\
& +2 \int_{0}^{\xi_{0}} \int_{0}^{\eta} c(\xi, \eta)\left(U^{(m)}-U^{(m-1)}\right)(\xi, \eta) d \xi d \eta \\
& +\int_{0}^{\xi_{0}} \alpha(1-\xi)\left(U^{(m)}-U^{(m-1)}\right)(\xi, \xi) d \xi \\
\geq & 0 \quad \text { in } \bar{D}_{\varepsilon}^{(1)},
\end{aligned}
$$

and thus, by induction,

$$
U\left(\xi_{0}, \eta_{0}\right)=\sum_{m=0}^{\infty}\left(U^{(m+1)}-U^{(m)}\right)\left(\xi_{0}, \eta_{0}\right) \geq 0 \text { in } \bar{D}_{\varepsilon}^{(1)} .
$$

(b) If condition (3.20) is satisfied, then it is easy to check that $U^{(1)}\left(\xi_{0}, \eta_{0}\right) \geq 0$ for any $\left(\xi_{0}, \eta_{0}\right) \in \bar{D}_{\varepsilon}^{(1)}$, and so, in view of (a), we see that $U\left(\xi_{0}, \eta_{0}\right) \geq 0$ for $\left(\xi_{0}, \eta_{0}\right) \in \bar{D}_{\varepsilon}^{(1)}$. Using the results of [11], we derive the following representation:

$$
U_{\eta_{0}}\left(\xi_{0}, \eta_{0}\right)=\int_{0}^{\xi_{0}} g\left(\xi, \eta_{0}\right) d \xi+\int_{0}^{\xi_{0}} c\left(\xi, \eta_{0}\right) U\left(\xi, \eta_{0}\right) d \xi
$$

and hence we conclude that $U_{\eta_{0}} \geq 0$ in $\bar{D}_{\varepsilon}^{(1)}$.

(c) If $g(\xi, \eta) \geq 0$ in $\bar{D}_{\varepsilon}^{(1)}$, then conditions (3.18) and (3.20) are obviously satisfied, and thus $U \geq 0$ and $U_{\eta_{0}} \geq 0$ in $\bar{D}_{\varepsilon}^{(1)}$. The conclusion $U_{\xi_{0}} \geq 0$ in $\bar{D}_{\varepsilon}^{(1)}$ follows from the fact that (see $[11])$

$$
\begin{aligned}
U_{\xi_{0}}\left(\xi_{0}, \eta_{0}\right)= & \alpha\left(1-\xi_{0}\right) U\left(\xi_{0}, \xi_{0}\right)+\int_{0}^{\xi_{0}}\left[g\left(\xi, \xi_{0}\right)+c\left(\xi, \xi_{0}\right) U\left(\xi, \xi_{0}\right)\right] d \xi \\
& +\int_{\xi_{0}}^{\eta_{0}}\left[g\left(\xi_{0}, \eta\right)+c\left(\xi_{0}, \eta\right) U\left(\xi_{0}, \eta\right)\right] d \eta
\end{aligned}
$$


In order to prove our results, we make use of the following proposition.

Proposition 3.3. Let $U(\xi, \eta)$ be the unique generalized solution for problem (3.13), where $c(\xi, \eta)$ and $g(\xi, \eta)$ are given by (3.14) and (3.15). Then $U(\xi, \eta) \in C^{n-1}\left(\bar{D}_{0} \backslash(1,1)\right)$ and $U(\xi, \eta) \geq 0$ in $\bar{D}_{0} \backslash(1,1)$; in addition $U_{\xi}(\xi, \eta) \geq 0, U_{\eta}(\xi, \eta) \geq 0$ in some neighborhood of the point $(1,1)$.

Proof. First note that in this case neither condition $g(\xi, \eta) \geq 0$ nor condition (3.20) is fulfilled. We will prove that condition (3.18) is satisfied. Introduce the polar coordinates $(\rho, t)$ and consider the function $g(\rho, t)=\rho^{1 / 2} E_{1}^{n}(\rho, t)$ in the domain $G_{0}=\{(\varrho, t): t>0, t<$ $\varrho<1-t\}$, then the representation formula (see (2.26))

$$
\frac{\partial}{\partial t} \rho^{1 / 2} H_{0}^{n}(\rho, t)=2(n-1) \rho^{1 / 2} E_{1}^{n}(\rho, t)=2(n-1) g(\rho, t)
$$

holds. Let $0 \leq \rho_{1} \leq \rho_{2} \leq 1$. Using (3.27), it is easy to see that, for the first approximation $U^{(1)}$ of the solution, one has (see (3.17))

$$
\begin{aligned}
2(n-1) & U^{(1)}\left(\frac{\rho_{1}+\rho_{2}}{2}, \frac{\rho_{2}-\rho_{1}}{2}\right) \\
= & \int_{\left(1+\rho_{1}\right) / 2}^{1} \rho^{1 / 2} H_{0}^{n}(\rho, 1-\rho) d \rho+\int_{\rho_{1}}^{\left(1+\rho_{1}\right) / 2} \rho^{1 / 2} H_{0}^{n}\left(\rho, \rho-\rho_{1}\right) d \rho \\
& -\int_{\left(\rho_{2}+\rho_{1}\right) / 2}^{\rho_{2}} \rho^{1 / 2} H_{0}^{n}\left(\rho, \rho_{2}-\rho\right) d \rho-\int_{\rho_{1}}^{\left(\rho_{2}+\rho_{1}\right) / 2} \rho^{1 / 2} H_{0}^{n}\left(\rho, \rho-\rho_{1}\right) d \rho \\
& +\int_{\left(1+\rho_{2}\right) / 2}^{1} \rho^{1 / 2} H_{0}^{n}(\rho, 1-\rho) d \rho+\int_{\rho_{2}}^{\left(1+\rho_{2}\right) / 2} \rho^{1 / 2} H_{0}^{n}\left(\rho, \rho-\rho_{2}\right) d \rho .
\end{aligned}
$$

Since $H_{0}^{n} \geq 0$, to prove that $U^{(1)} \geq 0$, it is enough to show that

$$
I=\int_{\left(1+\rho_{1}\right) / 2}^{1} \rho^{1 / 2} H_{0}^{n}(\rho, 1-\rho) d \rho-\int_{\left(\rho_{2}+\rho_{1}\right) / 2}^{\rho_{2}} \rho^{1 / 2} H_{0}^{n}\left(\rho, \rho_{2}-\rho\right) d \rho \geq 0 .
$$

For this purpose, we see that

$$
\begin{aligned}
I= & \int_{\left(1+\rho_{1}\right) / 2}^{1}(1-\rho) \rho^{-n+1 / 2}(2 \rho-1)^{n-3 / 2} d \rho \\
& -\int_{\left(\rho_{2}+\rho_{1}\right) / 2}^{\rho_{2}}\left(\rho_{2}-\rho\right) \rho^{-n+1 / 2} \rho_{2}^{n-3 / 2}\left(2 \rho-\rho_{2}\right)^{n-3 / 2} d \rho \\
= & \int_{\left(1+\rho_{1}\right) / 2}^{1}(1-\rho) \rho^{-1}\left(2-\frac{1}{\rho}\right)^{n-3 / 2} d \rho \\
& -\int_{\left(2+\rho_{1}-\rho_{2}\right) / 2}^{1}(1-\rho)\left(\rho+\rho_{2}-1\right)^{-1} \rho_{2}^{n-3 / 2}\left(2-\frac{\rho_{2}}{\rho+\rho_{2}-1}\right)^{n-3 / 2} d \rho .
\end{aligned}
$$

As a final step, notice that

$$
2-\frac{1}{t} \geq 2-\frac{\rho_{2}}{t+\rho_{2}-1} \geq 0 \quad \text { for } \frac{2+\rho_{1}-\rho_{2}}{2} \leq t \leq 1,
$$


and therefore $I \geq 0$. So, we conclude that condition (3.18) is satisfied. It follows now, by Theorem 3.1, that $U(\rho, t) \geq 0$ in $G_{0} \backslash(0,0)$. More precisely, for $\rho_{2}<\delta$, the last term in (3.30) is small enough for small positive $\delta$, and so

$$
I \geq \int_{3 / 4}^{1}(1-\rho)\left(2-\frac{1}{\rho}\right)^{n-1 / 2} d \rho:=c_{0}>0 .
$$

Thus, we find that $U^{(1)}(\rho, t) \geq c_{0}>0$ in a small neighborhood of the origin $(0,0)$. Therefore, for the solution $U(\xi, \eta)$ of problem $\left(\mathrm{P}_{\alpha, 2}\right)$ in coordinates $(\xi, \eta)$, it follows that $U(\xi, \eta) \geq U^{(1)}(\xi, \eta) \geq c_{0}>0$ in the corresponding neighborhood of the point $(1,1)$. Using the representation

$$
U_{\eta_{0}}\left(\xi_{0}, \eta_{0}\right)=\int_{0}^{\xi_{0}}\left\{g\left(\xi, \eta_{0}\right)+\frac{4 n^{2}-1}{4\left(2-\eta_{0}-\xi\right)^{2}} U\left(\xi, \eta_{0}\right)\right\} d \xi
$$

it is easy to see now that $U_{\eta_{0}}\left(\xi_{0}, \eta_{0}\right) \geq 0$ for $1-\delta \leq \xi_{0} \leq \eta_{0} \leq 1$ if $\delta>0$ is small enough. Furthermore, using the representation (3.26) of $U_{\xi_{0}}\left(\xi_{0}, \eta_{0}\right)$, we can prove an analogous result for $U_{\xi_{0}}\left(\xi_{0}, \eta_{0}\right)$.

Remark 3.4. In our opinion, the analogous result follows for all functions $E_{k}^{n}(\rho, t), k=$ $2,3, \ldots,[n / 2]-1$. As before, for $k>0$, the function $E_{k}^{n}(\rho, t)$ changes its sign in the domain, but due to the monotonicity of the solution $U(\xi, \eta)$, the desired result would follow. Also, by using the more general formula

$$
\frac{\partial}{\partial t} H_{k}^{n}(\rho, t)=2(n-k-1) E_{k+1}^{n}(\rho, t)
$$

this result could be obtained for $k>1$ too.

Now we are ready to prove Theorem 1.3 formulated in the introduction.

Proof of Theorem 1.3. We will find the desired lower estimates for the singular solution $u(\rho, \varphi, t)$ of problem (3.3), (3.4). For the corresponding right-hand side $g(\xi, \eta)$, defined by (3.15), set

$$
K=\int_{D_{1 / 2}^{(1)}} g^{2}(\xi, \eta) d \eta d \xi>0
$$

Let $\varepsilon \in(0,1 / 2)$ be fixed. Then, for the generalized solution $U(\xi, \eta)$ of problem (3.13), it follows that

$$
\begin{aligned}
0<K & \leq \int_{D_{\varepsilon}^{(1)}} g^{2}(\xi, \eta) d \xi d \eta \\
& =\int_{D_{\varepsilon}^{(1)}} U_{\xi \eta}(\xi, \eta) g(\xi, \eta) d \xi d \eta-\int_{D_{\varepsilon}^{(1)}} c(\xi, \eta) U(\xi, \eta) g(\xi, \eta) d \xi d \eta \\
& =: I_{1}+I_{2},
\end{aligned}
$$


where

$$
\begin{aligned}
I_{1}= & \int_{0}^{1-\varepsilon} \int_{\xi}^{1} U_{\xi \eta}(\xi, \eta) g(\xi, \eta) d \eta d \xi \\
= & \int_{0}^{1-\varepsilon}\left[U_{\xi}(\xi, 1) g(\xi, 1)-U_{\xi}(\xi, \xi) g(\xi, \xi)\right] d \xi \\
& -\int_{D_{\varepsilon}^{(1)}}\left(U_{\xi} g_{\eta}\right)(\xi, \eta) d \eta d \xi .
\end{aligned}
$$

In view of (3.15), it is obvious that $g(\xi, 1)=0$. Thus,

$$
I_{1}=-\int_{0}^{1-\varepsilon} U_{\xi}(\xi, \xi) g(\xi, \xi) d \xi-\int_{D_{\varepsilon}^{(1)}}\left(U_{\xi} g_{\eta}\right)(\xi, \eta) d \eta d \xi
$$

Since

$$
\begin{aligned}
\int_{D_{\varepsilon}^{(1)}}\left(U_{\xi} g_{\eta}\right)(\xi, \eta) d \xi d \eta= & \int_{0}^{1-\varepsilon} \int_{0}^{\eta}\left(U_{\xi} g_{\eta}\right)(\xi, \eta) d \xi d \eta \\
& +\int_{1-\varepsilon}^{1} \int_{0}^{1-\varepsilon}\left(U_{\xi} g_{\eta}\right)(\xi, \eta) d \xi d \eta \\
= & \int_{0}^{1-\varepsilon}\left[\left(U g_{\eta}\right)(\eta, \eta)-\left(U g_{\eta}\right)(0, \eta)\right] d \eta \\
& +\int_{1-\varepsilon}^{1}\left[\left(U g_{\eta}\right)(1-\varepsilon, \eta)-\left(U g_{\eta}\right)(0, \eta)\right] d \eta \\
& -\int_{D_{\varepsilon}^{(1)}}\left(U g_{\xi \eta}\right)(\xi, \eta) d \xi d \eta \\
= & \int_{0}^{1-\varepsilon}\left(U g_{\eta}\right)(\eta, \eta) d \eta+\int_{1-\varepsilon}^{1}\left(U g_{\eta}\right)(1-\varepsilon, \eta) d \eta \\
& -\int_{D_{\varepsilon}^{(1)}}\left(U g_{\xi \eta}\right)(\xi, \eta) d \xi d \eta,
\end{aligned}
$$

(3.38) becomes

$$
\begin{aligned}
I_{1}= & -\int_{0}^{1-\varepsilon}\left[U_{\xi}(\xi, \xi) g(\xi, \xi)+U(\xi, \xi) g_{\eta}(\xi, \xi)\right] d \xi \\
& -\int_{1-\varepsilon}^{1} U(1-\varepsilon, \eta) g_{\eta}(1-\varepsilon, \eta) d \eta+\int_{D_{\varepsilon}^{(1)}}\left(U g_{\xi \eta}\right)(\xi, \eta) d \xi d \eta .
\end{aligned}
$$

An elementary calculation shows that

$$
\begin{gathered}
g_{\xi \eta}(\xi, \eta)-c(\xi, \eta) g(\xi, \eta)=0 \\
g_{\xi}(\xi, \xi)=g_{\eta}(\xi, \xi)=\frac{1}{32(n-1)}(5-2 n)(1-\xi)^{n-7 / 2}<0 .
\end{gathered}
$$


332 New singular solutions of Protter's problem

By (3.40) and (3.36), it follows that

$$
\begin{aligned}
0<K \leq I_{1}+I_{2}= & -\int_{0}^{1-\varepsilon}\left[U_{\xi}(\xi, \xi) g(\xi, \xi)+U(\xi, \xi) g_{\xi}(\xi, \xi)\right] d \xi \\
& -\int_{1-\varepsilon}^{1} U(1-\varepsilon, \eta) g_{\eta}(1-\varepsilon, \eta) d \eta \\
& +\int_{D_{\varepsilon}^{(1)}} U(\xi, \eta)\left[g_{\xi \eta}-c g\right](\xi, \eta) d \xi d \eta .
\end{aligned}
$$

Thus, we see that

$$
\begin{aligned}
0<K \leq I_{1}+I_{2}= & -\int_{0}^{1-\varepsilon}\left[U_{\xi}(\xi, \xi) g(\xi, \xi)+U(\xi, \xi) g_{\xi}(\xi, \xi)\right] d \xi \\
& -\int_{1-\varepsilon}^{1} U(1-\varepsilon, \eta) g_{\eta}(1-\varepsilon, \eta) d \eta
\end{aligned}
$$

where, as it is easy to check,

$$
g_{\xi}(\xi, \xi)=\frac{1}{2}[g(\xi, \xi)]_{\xi}
$$

The function $U(\xi, \eta)$ is a classical solution of $(3.13)$ in $\bar{D}_{\varepsilon}, \varepsilon \in(0,1)$, with

$$
U_{\xi}(\xi, \xi)=\frac{1}{2}[U(\xi, \xi)]_{\xi}+\frac{1}{2} \alpha(1-\xi) U(\xi, \xi) .
$$

If we substitute (3.44) and (3.45) into (3.43), we get

$$
\begin{aligned}
K \leq I_{1}+I_{2}= & -\frac{1}{2} \int_{0}^{1-\varepsilon}[U(\xi, \xi) g(\xi, \xi)]_{\xi} d \xi-\frac{1}{2} \int_{0}^{1-\varepsilon} \alpha(1-\xi) U(\xi, \xi) g(\xi, \xi) d \xi \\
& -\int_{1-\varepsilon}^{1} U(1-\varepsilon, \eta) g_{\eta}(1-\varepsilon, \eta) d \eta \\
= & -\frac{1}{2}(U g)(1-\varepsilon, 1-\varepsilon)-\frac{1}{2} \int_{0}^{1-\varepsilon} \alpha(1-\xi) U(\xi, \xi) g(\xi, \xi) d \xi \\
& -\int_{1-\varepsilon}^{1} U(1-\varepsilon, \eta) g_{\eta}(1-\varepsilon, \eta) d \eta
\end{aligned}
$$

Note that $\alpha(\xi) \geq 0, g(\xi, \xi) \geq 0$, and according to Proposition 3.3, we have

$$
U(\xi, \eta) \geq 0 \quad \text { in } \bar{D}_{\varepsilon}^{(1)}, \quad U_{\eta}(1-\varepsilon, \eta) \geq 0 \quad \text { for small enough } \varepsilon>0 .
$$

Calculating $g_{\eta}(1-\varepsilon, \eta)$ and denoting

$$
1-\eta_{\varepsilon}:=\varepsilon \frac{(2 n-3)(2 n+1)-2 \sqrt{2(2 n-3)(2 n+1)(n-1)}}{4 n^{2}-1}:=\varepsilon n_{1},
$$

where the number $n_{1} \in(0,1)$, we find

$$
\begin{array}{ll}
g_{\eta}(1-\varepsilon, \eta)<0 & \text { for } 1-\varepsilon<\eta<\eta_{\varepsilon} \\
g_{\eta}(1-\varepsilon, \eta)>0 & \text { for } \eta_{\varepsilon}<\eta<1
\end{array}
$$


This, together with (3.46), implies that

$$
\begin{aligned}
K \leq & I_{1}+I_{2} \leq \int_{1-\varepsilon}^{\eta_{\varepsilon}} U(1-\varepsilon, \eta)\left|g_{\eta}(1-\varepsilon, \eta)\right| d \eta-\frac{1}{2}(U g)(1-\varepsilon, 1-\varepsilon) \\
& -\int_{\eta_{\varepsilon}}^{1} U(1-\varepsilon, \eta)\left|g_{\eta}(1-\varepsilon, \eta)\right| d \eta \\
\leq & U\left(1-\varepsilon, \eta_{\varepsilon}\right)\left[g(1-\varepsilon, 1-\varepsilon)-g\left(1-\varepsilon, \eta_{\varepsilon}\right)\right] \\
& -U\left(1-\varepsilon, \eta_{\varepsilon}\right)\left[g(1-\varepsilon, 1)-g\left(1-\varepsilon, \eta_{\varepsilon}\right)\right]-\frac{1}{2}(U g)(1-\varepsilon, 1-\varepsilon) \\
= & {\left[U\left(1-\varepsilon, \eta_{\varepsilon}\right)-\frac{1}{2} U(1-\varepsilon, 1-\varepsilon)\right] g(1-\varepsilon, 1-\varepsilon) }
\end{aligned}
$$

because $g(1-\varepsilon, 1)=0$. Moreover, since $g(1-\varepsilon, 1-\varepsilon)=\varepsilon^{n-5 / 2} / 8(n-1)$, we see that

$$
0<K \leq\left[U\left(1-\varepsilon, 1-\varepsilon n_{1}\right)-\frac{1}{2} U(1-\varepsilon, 1-\varepsilon)\right] c_{n} \varepsilon^{n-(5 / 2)}
$$

Using the fact that $U \geq 0$ and $U_{\eta} \geq 0$, we obtain

$$
\begin{aligned}
& 0<K \leq U\left(1-\varepsilon, 1-\tau \varepsilon n_{1}\right) c_{n} \varepsilon^{n-(5 / 2)}, \quad 0 \leq \tau \leq 1, \\
& 0<K \leq\left[U(1-\varepsilon, 1)-\frac{1}{2} U(1-\varepsilon, 1-\varepsilon)\right] c_{n} \varepsilon^{n-(5 / 2)} .
\end{aligned}
$$

For $\xi=1-\varepsilon, \eta=1$, we have $\varrho=t=\varepsilon / 2$ and (3.53) becomes

$$
0<K_{1} \varepsilon^{(5 / 2)-n} \leq u_{n}^{(2)}\left(\frac{\varepsilon}{2}, \frac{\varepsilon}{2}\right)-\frac{1}{2} u_{n}^{(2)}(\varepsilon, 0) .
$$

Finally, the inverse transformation gives

$$
u_{n}^{(1)}(\rho, \rho) \geq \frac{1}{2} u_{n}^{(1)}(2 \rho, 0)+K_{2} \rho^{-(n-2)} \geq K_{2} \rho^{-(n-2)},
$$

where the positive constant $K_{2}$ depends only on $n$. Analogously, (3.52) gives

$$
u_{n}^{(1)}\left(\rho, \frac{1-\tau n_{1}}{1+\tau n_{1}} \rho\right) \geq K_{2} \rho^{-(n-2)}, \quad 0 \leq \tau \leq 1 .
$$

Multiplying the function $u_{n}$ by $K_{2}^{-1}$, we see that

$$
\begin{gathered}
\left|u_{n}(\rho, \varphi, \rho)\right| \geq \frac{1}{2}\left|u_{n}(2 \rho, \varphi, 0)\right|+\rho^{-(n-2)}|\cos n \varphi| \geq \rho^{-n+2}|\cos n \varphi| \\
\left|u_{n}\left(\rho, \varphi, \frac{1-\tau n_{1}}{1+\tau n_{1}} \rho\right)\right| \geq \rho^{-(n-2)}|\cos n \varphi|, \quad 0 \leq \tau \leq 1
\end{gathered}
$$

hold, and then (1.13) follows. The proof of the theorem is complete. 


\section{Acknowledgments}

The authors would like to thank the referee for helpful suggestions. The essential part of the present work was finished while N. Popivanov was visiting the University of Ioannina during 2001 and 2002. N. Popivanov would like to thank the University of Ioannina for its hospitality. The research of N. Popivanov and T. Popov was partially supported by the Bulgarian National Science Fund under Grant MM-904/99 and by Sofia University under Grant 625/2002.

\section{References}

[1] S. A. Aldashev, Correctness of multidimensional Darboux problems for the wave equation, Ukrainian Math. J. 45 (1993), no. 9, 1456-1464.

[2] _ On Darboux problems for a class of multidimensional hyperbolic equations, Differential Equations 34 (1998), no. 1, 65-69.

[3] - Some problems for a multidimensional hyperbolic integro-differential equation, Ukrainian Math. J. 52 (2000), no. 5, 673-679.

[4] A. K. Aziz and M. Schneider, Frankl-Morawetz problem in $\mathbb{R}^{3}$, SIAM J. Math. Anal. 10 (1979), no. 5, 913-921.

[5] Ar. B. Bazarbekov and Ak. B. Bazarbekov, Goursat and Darboux problems for the twodimensional wave equation. I, Differential Equations 30 (1994), no. 5, 741-748.

[6] A. V. Bitsadze, Some Classes of Partial Differential Equations, Advanced Studies in Contemporary Mathematics, vol. 4, Gordon and Breach Science Publishers, New York, 1988.

[7] E. T. Copson, Partial Differential Equations, Cambridge University Press, Cambridge, 1975.

[8] D. E. Edmunds and N. I. Popivanov, A nonlocal regularization of some over-determined boundary-value problems. I, SIAM J. Math. Anal. 29 (1998), no. 1, 85-105.

[9] A. Erdélyi, W. Magnus, F. Oberhettinger, and F. G. Tricomi, Higher Transcendental Functions. Vol. I, McGraw-Hill, New York, 1953.

[10] P. R. Garabedian, Partial differential equations with more than two independent variables in the complex domain, J. Math. Mech. 9 (1960), 241-271.

[11] M. K. Grammatikopoulos, T. D. Hristov, and N. I. Popivanov, On the singularities of 3-D Protter's problem for the wave equation, Electron. J. Differential Equations 2001 (2001), no. 1, $1-26$.

[12] Singular solutions to Protter's problem for the 3-D wave equation involving lower order terms, Electron. J. Differential Equations 2003 (2003), no. 3, 1-31.

[13] L. Hörmander, The Analysis of Linear Partial Differential Operators. III, Grundlehren der mathematischen Wissenschaften, vol. 274, Springer-Verlag, Berlin, 1985.

[14] J. D. Jeon, K. C. Khe, J. H. Park, Y. H. Jeon, and J. B. Choi, Protter's conjugate boundary value problems for the two-dimensional wave equation, J. Korean Math. Soc. 33 (1996), no. 4, 857863.

[15] G. D. Karatoprakliev, Uniqueness of solutions of certain boundary-value problems for equations of mixed type and hyperbolic equations in space, Differential Equations 18 (1982), 49-53.

[16] S. Kharibegashvili, On the solvability of a spatial problem of Darboux type for the wave equation, Georgian Math. J. 2 (1995), no. 4, 385-394.

[17] K. C. Khe, Darboux-Protter problems for the multidimensional wave equation in the class of unbounded functions, Mat. Zamet. YAGU 2 (1995), no. 1, 105-109.

[18]_- On nontrivial solutions of some homogeneous boundary value problems for the multidimensional hyperbolic Euler-Poisson-Darboux equation in an unbounded domain, Differential Equations 34 (1998), no. 1, 139-142. 
[19] On the conjugate Darboux-Protter problem for the two-dimensional wave equation in the special case, Nonclassical Equations in Mathematical Physics (Novosibirsk, 1998), Izdat. Ross. Akad. Nauk Sib. Otd. Inst. Mat., Novosibirsk, 1998, pp. 17-25 (Russian).

[20] A. M. Nakhushev, A criterion for continuity of the gradient of the solution to the Darboux problem for the Gellerstedt equation, Differential Equations 28 (1992), no. 10, 1445-1457.

[21] N. I. Popivanov and T. P. Popov, Exact behavior of singularities of Protter's problem for the 3-D wave equation, Inclusion Methods for Nonlinear Problems. With Applications in Engineering, Economics and Physics (J. Herzberger, ed.), Computing Supplementa, Supplement 16, vol. 16, Springer-Verlag, New York, 2002, pp. 213-236.

[22] N. I. Popivanov and M. Schneider, The Darboux-problem in $\mathbb{R}^{3}$ for a class of degenerated hyperbolic equations, C. R. Acad. Bulgare Sci. 41 (1988), no. 11, 7-9.

[23] , On M. H. Protter problems for the wave equation in $\mathbb{R}^{3}$, J. Math. Anal. Appl. 194 (1995), no. $1,50-77$.

[24] M. H. Protter, New boundary value problems for the wave equation and equations of mixed type, J. Rational Mech. Anal. 3 (1954), 435-446.

[25] M. H. Protter and H. F. Weinberger, Maximum Principles in Differential Equations, PrenticeHall, New Jersey, 1967.

[26] K.-C. Tong, On a boundary value problem for the wave equation, Sci. Record (N.S.) 1 (1957), 277-278.

M. K. Grammatikopoulos: Department of Mathematics, University of Ioannina, 45110 Ioannina, Greece

E-mail address: mgrammat@cc.uoi.gr

N. I. Popivanov: Department of Mathematics and Informatics, University of Sofia, 1164 Sofia, Bulgaria

E-mail address: nedyu@fmi.uni-sofia.bg

T. P. Popov: Department of Mathematics and Informatics, University of Sofia, 1164 Sofia, Bulgaria E-mail address: topopover@fmi.uni-sofia.bg 


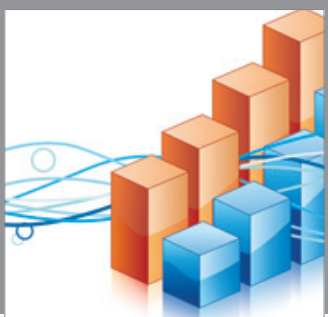

Advances in

Operations Research

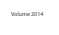

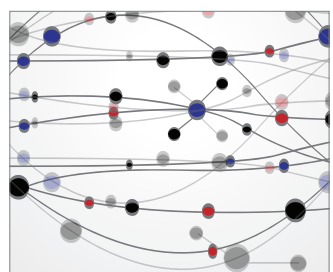

\section{The Scientific} World Journal
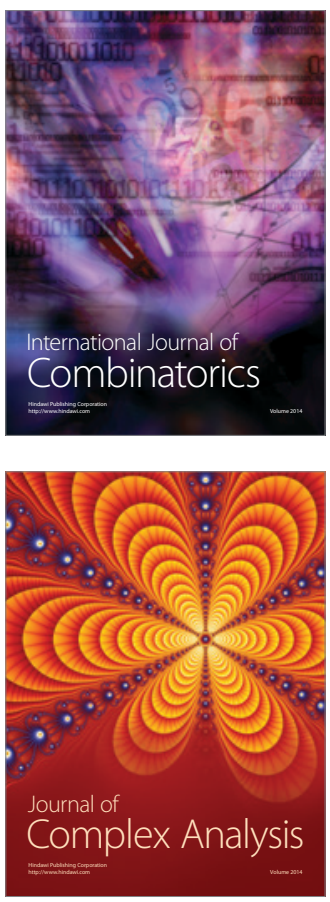

International Journal of

Mathematics and

Mathematical

Sciences
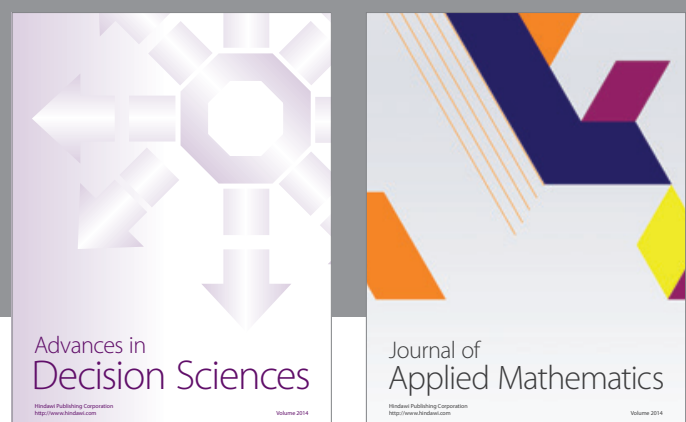

Journal of

Applied Mathematics
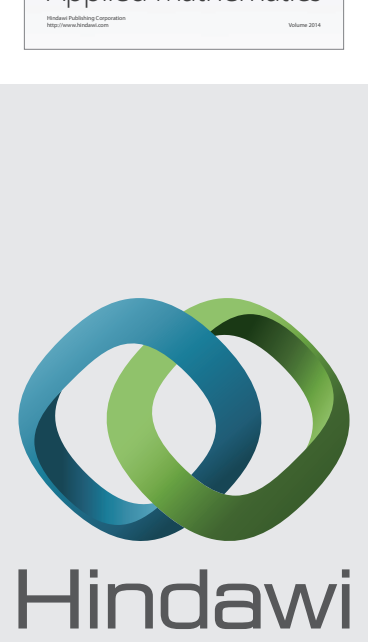

Submit your manuscripts at http://www.hindawi.com
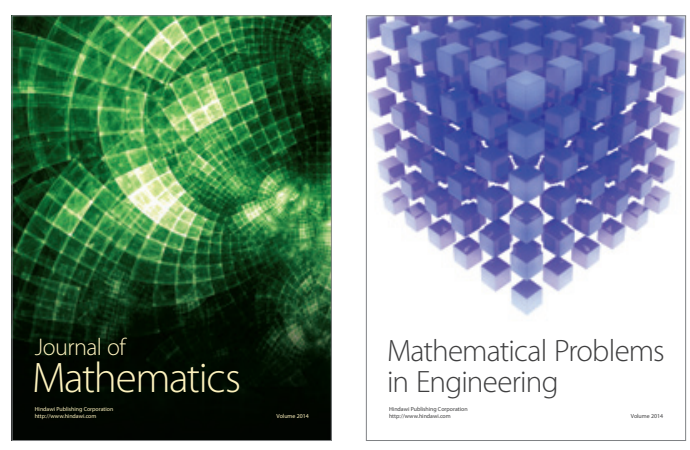

Mathematical Problems in Engineering
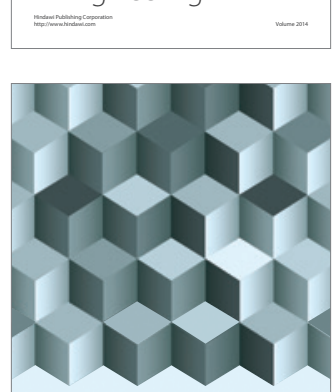

Journal of

Function Spaces
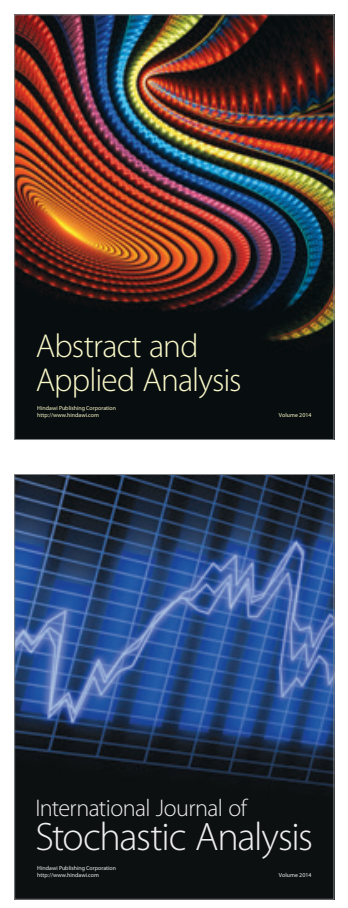

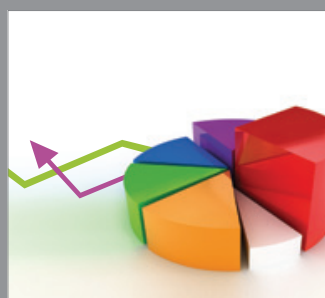

ournal of

Probability and Statistics

Promensencen
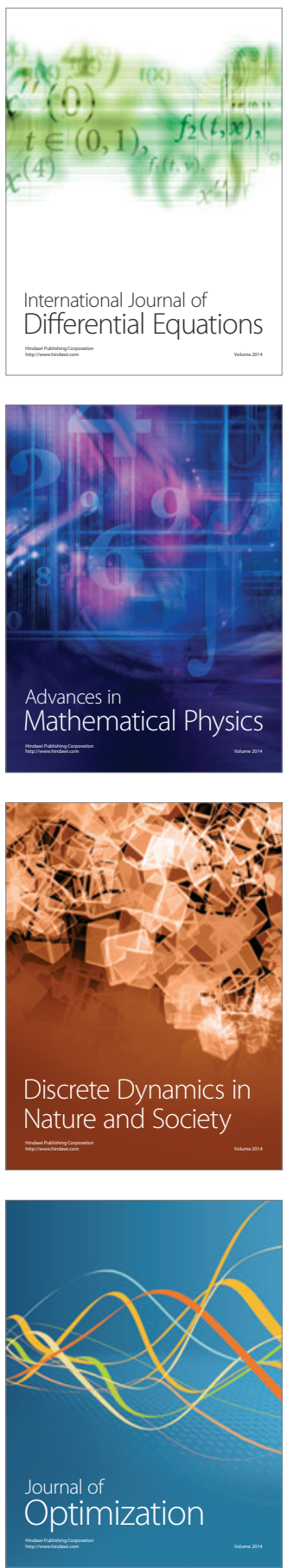